\title{
eSTIMe: Towards an All-in-One Geovisualization Environment for Daily Mobility Analysis
}

\author{
Aline Menin \\ Univ. Grenoble Alpes, CNRS, Grenoble INP, LIG \\ Grenoble, France \\ aline.menin@univ-grenoble-alpes.fr
}

Paule-Annick Davoine

Univ. Grenoble Alpes, CNRS, Science Po Grenoble, PACTE and Univ. Grenoble Alpes, CNRS, Grenoble INP, LIG

Grenoble, France

paule-annick.davoine@univ-grenoble-alpes.fr

\author{
Sonia Chardonnel \\ Univ. Grenoble Alpes, CNRS, Science Po Grenoble, PACTE \\ Grenoble, France \\ sonia.chardonnel@univ-grenoble-alpes.fr \\ Luciana Nedel \\ Institute of Informatics \\ Federal University of Rio Grande do Sul \\ Porto Alegre, Brazil \\ nedel@inf.ufrgs.br
}

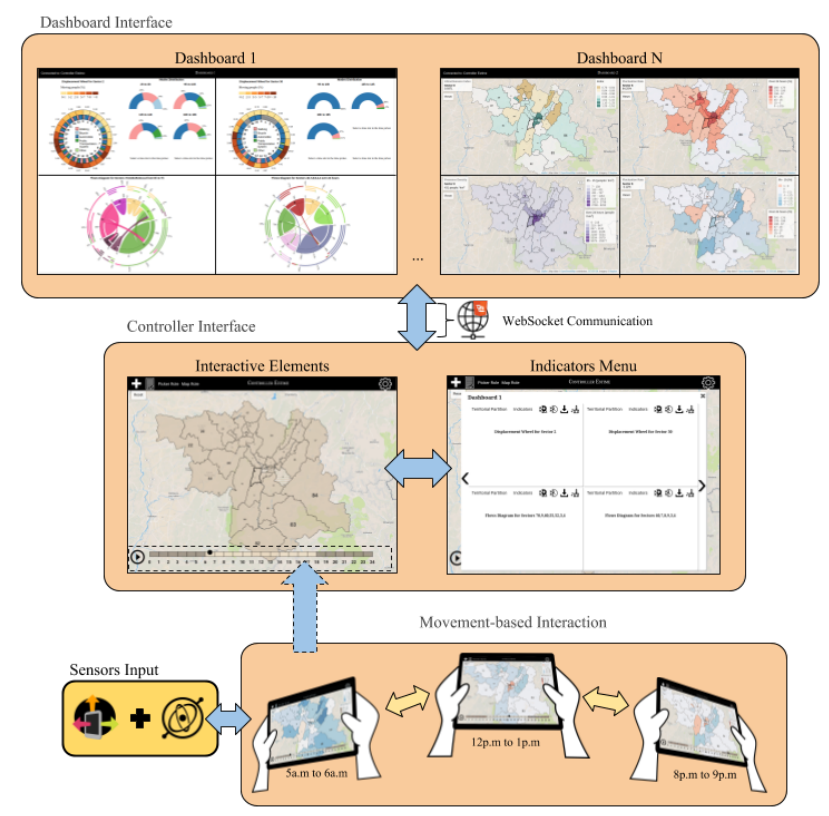

\begin{abstract}
Daily mobility data describes individual displacements over 24-hours periods and are an important source of information to understand the real rhythm of a city, to provide appropriate transportation policies, and to support investment decisions. Geovisualization researchers have designed multiple coordinated views environments, combining spatial and temporal dimensions, and providing indicators comparison. Daily mobility analysis is complex and requires simultaneous exploration and combination of different indicators at different spatial and temporal granularity levels. The design of effective geovisualization environments supporting this analysis evokes several challenges due to the diversity and multiplicity of indicators, the granularity of space and time, and time integration. In this paper, we propose a geovisualization approach enabling the dynamic visualization of diverse indicators, as well as the exploration of space, time, and other attributes. We use multiple screens embedding customizable dashboards and allowing the users to arrange views and compare indicators as it better fits their analysis. It also integrates a mobile device serving as a display and interaction tool to physically control the evolution of the visualization on time.
\end{abstract}

\section{INTRODUCTION}

The study of the forms of settlements and the organization of individuals' activity programs in space and time is important to understand and guide the development of better urban environments. In the past few years, the rise of tracking technology enabled the collection of different sorts of data describing the population's movements within a territory. The spatiotemporal Triad scheme [1] is commonly used to analyze this movement data since it enables the exploration of entities of interest in time and space at different levels of aggregation.

Urban mobility data can be studied on the basis of three entities: the flows and displacements, the territory settlement dynamics, and the individual trajectories. While the flows describe the number of displacements between two spatial locations, the territory settlement dynamics consider the presence and mobility of the population. Individual trajectories describe the sequence of activities performed by someone in space and time. The analysis of these entities rely on the derivation of indicators that can be used to provide information
Fig. 1. Our approach comprises customizable dashboards (top) embedded in a multi-screen environment. A tablet holds the main interface (middle), which interacts with the dashboards and support our movement-based technique (bottom) for time animation.

to support decision-making for transportation policies and investments; as a basis for comparisons among metropolitan areas, and; as a sense of whether system performance is improving or getting worse [2].

Along with the emergence of geovisualization techniques, geographers began to work with computer scientists for developing conceptual frameworks and methodologies based on a visual and exploratory analysis of spatiotemporal data [3]. These solutions often comprise dashboards with numerous interactive and coordinated views with graphics and cartographic representations of the data. It enables the interaction with multiple dimensions of the represented phenomenon to 
conduct spatial, temporal, and spatiotemporal analyses.

The design of existing geovisualization environments is frequently driven by specific problems, such as traffic jam detection [4], usage patterns of shared bicycles systems [5], social segregation in metropolitan regions [6], and life or individual trajectories analysis [7]-[9]. It would use data sources suitable to answer the problem-related questions, which might not require information on activity programs, for instance. It likely to favor only one form of representation, which is centered either on the territory settlement dynamics (e.g., through animated maps [10]), or the individual's movements (e.g., visualizing trajectories in 3D through the space-time cube - STC [11]). To the extent of our knowledge, there is not an unified environment (free and accessible) that gives the user the possibility to, simultaneously, explore the indicators summarizing aspects of displacements and flows, and to analyze and visualize the mobility dynamics at the individual and population levels.

The design and development of such unified environment evokes multiple challenges, such as conceiving visualization techniques suited to represent urban mobility indicators and to define interaction methods to handle the dialogue between space and time, while exploring the data at different levels of aggregation. This process should also consider the reasoning mechanisms supporting the visual analysis, which are associated with a cognitive process, resulting in different ways to which users perceive the visual representations. Hence, the proposed environment should be evaluated to guarantee its effectiveness, efficiency, and user satisfaction [12].

Our approach aspires the design and development of a geovisualization tool for urban mobility analysis, such as the one described above. We propose a multi-screen environment composed of customizable dashboards and a movement-based interaction technique for controlling the temporal evolution of the data. A tangible interface supported by a mobile device was designed to control space and time, and a novel visualization technique (the mobility wheel) based on a circular doublering donut diagram was proposed to represent the mobility practices of a population (see Fig. 1). We also present the development and evaluation of a prototype that supports the spatiotemporal analysis of flows and displacements and the territory settlement dynamics.

The remainder of this paper is organized as follows. Section II presents previous works on visual solutions for urban mobility data; analytical dashboards, and the use of mobile devices on information visualization. Section III presents the specifications that guided our proposal while Section IV details the eSTIMe prototype. Sections V and VI present the pilot study and the first impressions of users, and the evaluation process we used. Finally, Sections VII and VIII discuss our results, limitations, conclusions and future work.

\section{RELATED WORK}

Studies on urban mobility have increased in the last years, especially due to the diversity of data sources available, providing information on individuals' movements. In addition to the visualization tools mentioned earlier, Shi et al. [10] visually explore mobility metrics to identify vibrancy, fluidity, commutation, and diversity patterns. $\mathrm{Lu}$ et al. propose a ranking-based visual analysis method to explore vehicles travel behavior [13], and use taxis trajectories to visually explore origin-destination (OD) patterns [14] .

Since the analysis of the public transportation system (PTS) passengers' behavior is valuable for transportation researchers and urban planners, visual solutions were designed to explore this data [15], [16]. Zeng et al. [17] analyze the impact of mobility-related factors (riding, transfer and waiting time), and their temporal variations on the shape of passenger mobility.

Twenty-five years ago, bike-sharing systems were designed and today's technology provides usage data serving as complementary information to understand urban mobility. Shi et al. [5] propose a visual analytics system: to advice for optimal bicycle dispatch via stations categorization based on their main functions; to explore the spatial direction of biking moving via flow correlations and differences among multiple stations; and to visualize the influence of multiple factors on bicycle rental numbers. Latent user activity patterns in bike-sharing and their comparison in/between cities is also possible [4].

Ma et al. [18] visually explore flow crowd volumes, directional dynamics, and spatiotemporal patterns to inspect human social relations and their physical movements, while Zeng et al. [19] propose an interactive points-of-interest (POI) mobility signature to illustrate how human mobility varies over POIs in different categories and across different areas. Techniques like Voronoi tesselations and stacked elliptical activity glyphs are employed to visualize activity regions and to detect urban regions sharing similar activity patterns [20].

These studies share the goal of exploring urban mobility patterns. Their conception is often founded on hypotheses, and they use databases describing mobility mostly through spatiotemporal data, without taking into account attributes that could explain why and how people move. Understanding the reasons for people's displacements and their modes of transportation is as relevant for understanding the urban mobility dynamics as for describing their trajectories in space and time. Therefore, the relevance of an environment that enables the exploration of every dimension of the data and their internal relations is evident.

Faceted analytical dashboards are "a set of interactive charts (primarily graphs and tables) that simultaneously reside on a single screen, each of which presents a different view of a common dataset, and is used to analyze that information" [21]. These powerful decision-support tools applied to geographic information visualization have been proved to increase the spatial thinking performance, and may also improve the user performance [22].

Chitarro [23] discusses the challenges and advantages of visualizing information on mobile devices' screens, such as the usefulness of mobile-map applications for navigation and interaction. The transfer of multiple coordinated views (MCV) visualizations from normal-size monitors to large displays is a growing practice. Nonetheless, this requires new interaction 
techniques, since using the traditional mouse might be neither possible nor efficient. Langner et al. [24] and Kister et al. [25] propose sets of interaction techniques for large displays using mobile devices. Pahud et al. [26] use the mobile device as a sort of portal through which the user can explore, interact, and annotate n-dimensional data visualizations. Pietroszek et al. [27] leverage the tilting movement of a mobile device to interact into a 3D space by "casting" the 2D plane of the device. Besanon et al. [28] use complementary tangible and tactile inputs to achieve common $3 \mathrm{D}$ visualization tasks.

\section{Design RATIONALE}

\section{A. Assumptions}

As stated by LeRoux et al. [6], the rhythm of a city can change from day to nighttime. They studied the social composition of different neighborhoods over 24-hours and verified that it is similar during the night, but it evolves during the daytime, as the most favored people would move towards areas providing more local resources, while the disadvantaged people would be retained at their residential neighborhood. Therefore, the study of urban mobility through individuals' daily activity-based trips can assist public agents, e.g., urban planners, on targeting areas to implement interventions more connected with the real rhythm of the city.

Daily mobility data describes the movements of individuals throughout a studied territory along with 24-hours. It contains information on displacements performed to achieve a set of goals, such as going to work, to the school, or visiting someone. These goals describe people's motivations to move from one place to another, as well as their activities when staying at their final spatial location. In this context, an individual trajectory consists of a sequence of stops and moves. The former described a time interval when the individual was present in a spatial location performing a specific activity, while the latter represents a spatiotemporal line defined by two locations representing either two consecutive stops or a trajectory beginning or end [29].

The mobility indicators should be easy to interpret, presenting the actual situation through a representative picture, and enabling comparisons with other indicators [30]. Data exploration from the earlier mentioned entities of interest relies on the derivation of complementary indicators. For instance, indicators summarizing the flows and displacements can help to understand the influence of population migrations on a territorial settlement. The analysis of individual trajectories through the activity-based approach [31] allows to identify and characterize complex travel behavior, as well as extreme profiles such as hyperactive and stationary individuals.

Many areas within urban policies benefit from daily mobility analysis, supporting decisions on transportation offers, accessibility, air quality control, public health, and wellbeing. Thus, numerous experts (e.g., researchers, diagnostic managers, local authorities) have to manipulate mobility data to extract knowledge from it, without being transportation specialists. They compose the profile of the end-users of our geovisualization environment. Thus, our proposal should enable these users to:

- describe the daily mobility according to three entities of interest: the flows and displacements, the territory settlement dynamics, and the individual trajectories

- analyze and compare complementary indicators in the aggregate and individual levels

- explore the temporal dynamics of indicators to identify the daily rhythms of the city and its population

- compose the analytical dashboard according to their needs, choosing the indicators, spatial zones and periods

\section{B. Our Approach}

The integration of time and space dimensions is itself a challenge on cartographic and geovisual representations. Smallmultiples and animation are the foremost used techniques to dynamically explore time. The first technique displays the information changes side-by-side via time slices, which suffers from spatial limitation and resolution since each slice reduces as their number increases. The second option can be thought as a movie presenting the information as time slices one after another in a pre-defined order. Though animation does not suffers from low spatial resolution, fast animations can be difficult to understand, since the user must rely on their memory to understand the data [32].

The interaction method proposed by us to explore time relies on the animation concept. While we still present the information changes in the form of consecutive time slices, we allow the user to physically control it by tilting a tablet.Our inspiration comes from the use of mobile devices to interact with visualizations and 3D applications [33], and the advantages of using it as a second screen [34]. Intensive bodily interaction is known to be more engaging [35], and proprioception (i.e. the sense of self-movement and body position) could help the user to retain information by using the position and orientation of their hands and wrists as a recall reference. More than interacting with the temporal dimension, the mobile device also serves as a second screen holding map-based views and a tactile interaction space to manipulate indicators and views.

Analytical dashboards are largely used in visualization, being designed through a set of defined and effective views to achieve the user's goals. In this work, we want to give the users a certain freedom so they can custom the dashboard as they judge suitable to their analysis. Believing that larger workspaces could improve the analysis, we adopt customizable dashboards embedded into a multi synchronized screens environment. Further, this approach provide enough space to compare indicators at different spatiotemporal granularities.

As a proof of concept we developed eSTIMe (Fig. 1), a prototype to explore daily mobility data in the aggregate level.

\section{ESTIME}

\section{A. Data Description}

eSTIMe aims to support different datasets describing daily mobility, regardless of their sources. Currently, we use the data from a Household Travel Survey (HTS), since it provides 
information on individual displacements in space and time, including their motivations and transportation modes. This enables us to derive the individual activity-based trips and to aggregate presence and mobility of the population at different spatial locations and time intervals, as well as to derive the volume of flows between different locations. We recovered the 2010 edition [36] of a survey regularly carried out in the region of Grenoble, France, since 1976.

The data includes the displacements performed by all household members aged five and older during 24-hours, from 4am (the day before) to 4am (the survey day). It details the departure and arrival location and time, transportation mode and motivation for each displacement. The locations are described by places (pulling sectors defined in the survey), and the temporal dimension is discretized into one-hour intervals. The survey provides data on about 63,000 trips performed on weekdays from 7,600 households. We provide a diverse spatial granularity through three territorial partitions (97, 39, and 12 sectors); they all contain smaller surfaces in inner Grenoble and larger ones in the mountain area.

\section{B. Map View}

Indicators summarizing population's presence in different locations are visualized through a choropleth map, a wellknown technique used for geographical data representation. We derived indicators of presence density per location, i.e. present persons per square kilometer; fluctuation measures the rising and falling of population along the day through the difference between people present and the sector population, and; attractiveness, which identify whether the real density of a sector is equivalent to the population density [37]. Since presence density and fluctuation can be explored over 24 hours and per time interval, their maps have two legends: one explains the values for the current time interval, and the other the values for the whole day. Fluctuation values represent population gain and lost. Attractiveness indicates equilibrium between real and population density as 1 (values over 1 indicate greater real density and under otherwise). Fluctuation and attractiveness are depicted through diverging color scales, while density uses a linear color scale.

\section{Mobility Wheel}

Indicators summarizing the mobility practices of a population complement the presence dynamics. They reveal active persons' routines along the day through the number of people moving from one place to another at each time interval and the distribution of these movements according to different transportation modes. For each sector, the indicator represents the number of persons moving towards this location, and the transportation modes used to reach this destination.

Timewheels are often used to represent time-series data [38], which inspired the design of our Mobility Wheel (Fig. 2). We adapted the view of a double-ring donut chart, forming each ring with 24 rectangles symbolizing 24-hours. Each time interval on rings display: in the outermost, people in movement per sector's population; in the innermost, the partition of
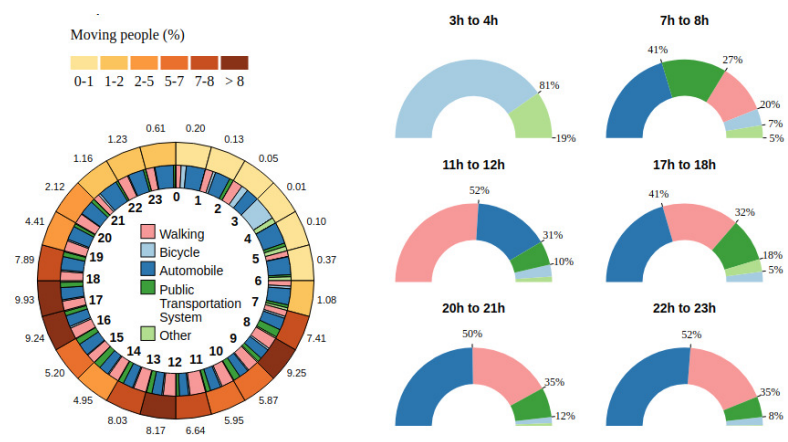

Fig. 2. The Mobility Wheel displays the movement patterns of active people, while the arc charts compare the usage of transportation modes across time intervals.

movements per transportation mode. Arc charts displayed sideby-side next to the wheel allow to zoom into the transportation modes distribution, and to compare them across 6 time intervals. This view helps to identify the mobility patterns of the population over the day at a glance and to understand how they move around the territory by matching the intensity of movement and the transportation modes they engage.

\section{Flows View}

Representing flows without losing the spatial component and avoiding occlusion is a challenging task [39]. Flow maps are largely used to represent the volume of flows within a territory, but they have constraints on the design of legible maps. Therefore, we adopted the origin-destination (OD) pair relationship view introduced by Shi et al. [5] to explore OD flows between spatial locations. It uses a chord diagram, where arcs represent spatial locations. The arcs are connected by ribbons, which thickness describe the flows volume exchanged between the locations. The ribbon color determine the flow origin. We provide spatial information virtually binding the chord diagram to the map view on the tablet display. Flows filtering is available by selecting a location on the map. The indicator can be explored over 24 hours or per time period. The view can display flows exchanges within the entire territory, or the user can choose a set of up to 10 locations (for the sake of readability).

\section{E. Interaction}

eSTIMe supports regular interactions such as pan and zoom, as well as spatiotemporal filtering. The angles produced via the tablet tilting are mapped to slots in the time picker. Since the daily mobility events generate everyday patterns, a cyclic arrangement (timewheel) seems appropriate. The existing environments often arrange time linearly in a timeline. Our interface, for the other hand, provides both formats upon choice. The user would tilt the tablet either counter- and clockwise for selecting time intervals in the timewheel, or from left to right and contrariwise in the timeline.

The main interface, so called Controller, provides a menu to choose the indicators and customize them under different spatiotemporal granularities before displaying them on 
a dashboard. It can hold multiple customizable dashboards. By default, each dashboard comprises four blank windows, progressively fulfilled according to the user's needs. Time and space are explored via a time picker and a map available on the Controller. The former is used to change time and to open arc charts next to Mobility Wheels. The map view supports pan and zoom; selection of locations to generate the Flows View or the Mobility Wheel; and spatial filtering on the Flows View. In case our prospect users want to create reports of their exploration, we also provide them with the possibility to download the views as an image.

\section{F. Implementation Details}

The basic setup of eSTIMe consists on instantiate it twice: one instance holds the main interface and should be displayed on the tablet, while the second one comprises a dashboard, where the views are displayed and organized. It should be displayed on a large screen for a better experience. The prototype is a Web-based application. A server was developed in Java and uses WebSocket technology to manage the communication between the Controller and the dashboards. The client was developed in JavaScript and uses D3 (DataDriven Documents) and Leaflet libraries to generate charts and cartographic representations, respectively. The raw data was pre-treated through $\mathrm{R}$ scripts, and locally stored as geojson for geographical information, json for the flows data, and csv for the remaining.

\section{Pilot Study And Redesign}

A first prototype was tried out by 13 volunteers aged 36 years old in average $(S D=10)$. This trial verified whether the prototype was usable and could assist users on answering questions on daily mobility analysis. Since the interface comprises two time pickers, we also assessed the users reaction to our movement-based time interaction. We followed a withinsubjects study design to test two conditions: timeline and timewheel. Participants were asked to answer two sets of 6 questions, one for each variable. The questionnaire contained simple (e.g. which is the presence density in sector 5 between $7 \mathrm{am}$ and 8am?) and complex (e.g. where are people coming from in the 3 most attractive sectors?) questions. We measured users self-reported workload for each condition through the NASA Task Load Index (TLX) [40], a multi-dimensional rating procedure which provides an overall workload score based on a weighted average of ratings on six sub-scales: Mental Demand (MD), Physical Demand (PD), Temporal Demand (TD), Own Performance (OP), Effort (EF), and Frustration (FR). A post-test questionnaire collected users' subjective assessment on usability through the SUS questionnaire [41], and their preferences over the time arrangements.

For the sake of reproducibility, the output data of the user studies and the scripts treating it are available as supplemental material. Data was submitted to a mixed design with the experimental conditions as within-subjects and the user profile as a between-subjects test. We performed a Shapiro-Wilk Normality test to verify if the data comes from a normal distribution and a Fligner-Killeen test to verify if the variances in each group are the same. When the data passes both tests we performed an One-Way ANOVA. Otherwise, we submitted the data to non-parametric tests, Friedman for paired and Kruskal-Wallis for unpaired groups. When finding statistical significance in multiple variables comparisons we ran Tukeys range test for One-Way ANOVA, and Nemenyi test for the others. We analyzed whether variables were related through Pearson correlation.

Response time and accuracy were similar in both conditions: around 26 minutes and $86 \%$ of correct answers. These variables are $56 \%$ correlated in the timewheel condition $(p=$ 0.058). Both timeline and the timewheel induced a mean selfreported workload of 63 and were high mentally demanding. In the timewheel, the MD was significantly stronger than PD, TD, and FR $(p<0.001)$. For both conditions, PD was lower than EF and OP $(p<0.001)$. The timeline was found less frustrating than effortful $(p<0.001)$. The more mental demanding the task, the less physical effort it requires $(R=0.6, p<0.05)$. Though PD received the lowest scores, it seems to worsen response accuracy as it increases $(R=0.69$, $p<0.001)$. Mental demand appears to reduce users' selfperceived performance $(R=-0.83, p<0.001$ for the timewheel, and $R=-0.51, p=0.078$ for the timeline), yet it does not seem to affect response accuracy.

Older participants took longer to finish the experiment in the timewheel condition $(R=0.72, p<0.01)$. In the same way, the mental demand is higher for older participants in the timeline $(R=0.65, p<0.05)$, and it appears to happen also in the timewheel $(R=0.5, p=0.085)$. Great prior knowledge on the territory mobility also increased mental demand $(R=0.58, p<0.05)$ in the timeline. The longer the response time, the less frustrated users felt $(R=0.58$, $p<0.05)$. We suppose users got comfortable with the prototype, decreasing frustration feelings. The usability of our prototype was assessed on $48 / 100(S D=8.66)$. The timeline was favored over the timewheel $(M=3.53, S D=1.19$ in a 5points Likert scale). Users are more familiar to it and the lower degrees-of-freedom make the timeline easier to manipulate.

Users provided satisfactory answers to the proposed questions in the pilot study, yet their response time was longer than desirable for everyday use and they reported high workload scores. The interface lacked on intuitiveness, specially regarding interaction with dashboards. To display a view, the user should select the territorial partition, the indicator, the dashboard and submit the choices. The view would be displayed in the next blank window, re-assorting the views each time the user deleted or added something. We then transformed it into a slider menu interface, where each slide corresponds to a dashboard, holding four "windows" as in the dashboard interface, and the user can directly control where and how the indicators are viewed (Fig. 3A). The charts supporting the analysis and comparison of modes of transport used by active people were designed as bar charts, which was hard to understand at a glance. We modified it into arc charts (Fig. 3B), following the color code of the 

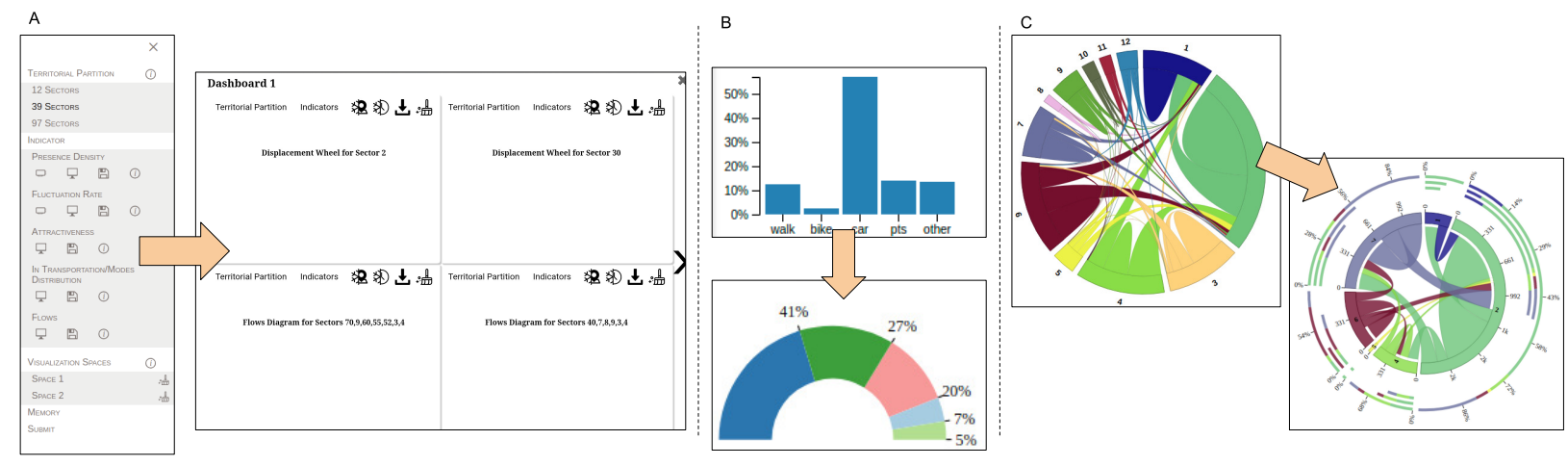

Fig. 3. Main modifications on the prototype after the pilot study results. The menu (A) have been remade into a more intuitive interface. The charts displaying the distribution of transportation modes (B) were changed from bars to arc, which also link the modes to their respective colors. The Flow View (C) was upgraded into a more intuitive and informative diagram.

wheel's inner ring, fastening information assimilation. We have represented flows exchanges through a simple chord diagram, which was hard to understand since flows direction was only pointed by ribbons color and it did not provide information on flows volume. We upgraded it to a more legible and intuitive diagram [5] (Fig. 3C).

\section{USER EVALUATION}

The new version of eSTIMe was submitted to a two-part evaluation (below): to assess the movement-based interaction technique; and to evaluate effectiveness with prospect users.

\section{A. Experiment I: Time Animation}

Seventeen unpaid persons, researchers and $\mathrm{PhD}$ students, aged 33 years old in average $(S D=11.18)$, took part in a within-subjects experiment to compare 4 conditions: timewheel (A) or timeline (B) controlled by tilting, and traditional animation controlled by mouse (C) or touch (D), hypothesizing the (H1) animation performance is similar with mouse and touch interactions; (H2) tilting movement provides better answers than the traditional interaction; and (H3) movementbased interaction is preferred over the mouse.

We gave the participants four (one per condition) 3questions sets regarding different conditions (either presence density or fluctuation rate) and spatial locations $(\mathbf{x}, \mathbf{y}, \mathbf{z})$ : how the variable vary along the day in sector $\mathbf{x}$ ?; which are the periods in sector $\mathbf{y}$ when the variable value decreases?; which are the periods in sector $\mathbf{z}$ when the variable value increases? Subjective usability of each technique was assessed via UMUX-Lite questionnaire [42]. The post-test questionnaire gathered users' preferences over the proposed techniques through a pair-wise comparison and a 4-item rating assessing the agreeableness of each technique in a 5-point Likert scale.

Response time was around 6 minutes for each experimental condition, progressively decreasing from the first to the last question $(p<0.01)$. Condition A (timewheel) provided a $50 \%$ response accuracy, significantly lower than the other conditions (around 85\%, $p<0.05$ ). Overall, users preferred to control animation via touches. From the pair-wise comparison, conditions B (linear movement) and C (traditional animation) were similarly appreciated, and condition D is preferred over C. The assessed usability was not significantly different between the techniques (42/100 in average). Users found the timewheel was the most frustrating and difficult to use. As expected, the traditional animation was considered easy to use, since it is well-known for time exploration and users are more familiar with mouse interaction.

Participants' prior experiences on non-conventional interaction appear to increase response accuracy $(R=0.35$, $p=0.013)$ in condition $\mathrm{D}$, and to reduce response time in condition $\mathrm{B}(R=0.28, p<0.05)$ and $\mathrm{D}(R=0.24$, $p=0.087$ ). Similar to the pilot study, older people provided less accurate answers $(R=-0.29, p<0.05$, condition $\mathrm{D})$, and took longer to finish $(R=0.35, p<0.05$, condition $\mathrm{A})$ it.

The cyclic tilting movement was found unnatural and the rectangles in the timewheel too small, challenging the pointer's placement. The timewheel takes about $25 \%$ of the screen's height for the sake of the map readability, which became a limitation in our approach, since we use a 9.7-inch screen tablet. Participants found hard to interpret the fluctuation map because of its diverging color scale and the proportional values (to the population). The legends changing at each time interval disturbed some participants.

In summary, since we have found similar response times and accuracy between interactive animation via mouse and touches, we accept H1. Response accuracy was lower while using the cyclic movement, but linear movement provided similar response accuracy and time as the traditional animation. We reject $\mathbf{H 2}$, because performance with the movementbased technique was not better, but rather similar to traditional animation. However, users enjoyed the linear movement, as well as the tangible animation, finding it easy to use. We accept $\mathrm{H3}$ and consider to merge both techniques to enable the user to play the animation via device tilting and to select time intervals via touch.

\section{B. Experiment II: The Environment}

Twenty-four volunteers (21 valid), aged 22 years old in average $(S D=1.82)$, students with satisfactory knowledge on 
the target domain took part in this experiment. We followed an one-group posttest-only design to evaluate the effectiveness of our environment on assisting the analysis of daily mobility data. Participants in the pilot study mentioned one need a longer training time to master the interface. Therefore, we designed it as a two-part experiment. The first part comprised two 3-hours sessions in which users worked in pairs to answer a set of 16 questions. In these sessions, a PC monitor displayed the Controller, and a mouse supported the interaction.

The experimental (collective) sessions took place two weeks later, where 12 participants worked in parallel to answer 14 analytic questions. An automatic training phase was performed through 16 interaction-related questions. A post-test questionnaire included the SUS and a reduced version of the NASA TLX, containing only the ratings part in a 10-point scale. Scores for each sub-scale were determined as the average rating times 10, and the general score was the mean score of sub-scales. It also measured users' preference on using a PC monitor or a tablet as second screen.

Assessed usability is still moderate (40/100), while selfreported workload decreased $(M=33.3, S D=6.8)$. Users strongly scored MD $(M=41.4, S D=9.10)$ and $\mathrm{OP}$ $(M=54.3, S D=9.78)$. However, no correlation was observed between OP and neither response time nor accuracy. Responses were $81 \%$ accurate, varying across spatial locations and indicators. The responses regarding the $4^{\text {th }}$ location were less accurate than the remaining $\left(p<0.001\right.$ against the $2^{\text {nd }}$, and $p<0.05$ against the $1^{\text {th }}$ and $3^{\text {rd }}$ ). Accuracy on questions about attractiveness was higher than presence density $(p<0.05)$, which also got less correct answers than flows $(p<0.001)$. The latest was also more accurate than fluctuation rate $(p<0.01)$ and population mobility $(p<0.001)$.

Response time was about 35 minutes $(S D=12.9)$, with an average of 2.49 minutes $(S D=2.26)$ per question. Small correlations suggest response time appears to increase FR $(R=0.12, p<0.05)$, EF $(R=0.16, p<0.01)$, and $\mathrm{PD}$ ( $R=0.2, p<0.001)$, and to reduce accuracy $(R=-0.12$, $p<0.05)$. Participants' prior knowledge on mobility appears to increase OP $(R=0.49, p<0.001)$, and total workload $(R=0.26, p<0.001)$. More experienced on nonconventional interaction users reported higher PD $(R=0.3$, $p<0.001)$, MD $(R=0.34, p<0.001)$, and total workload ( $R=0.2, p<0.001)$. However, it does not seem to affect response time and accuracy. Users enjoyed the tablet as a second screen $(M=3.95, S D=1.43)$ and preferred using it rather than a PC monitor $(M=4, S D=1.22)$, since they could display the views on a large screen while interacting on the tablet, which facilitates data exploration.

\section{DISCUSSION}

We designed and developed a multi-screen geovisualization environment embedding customizable dashboards to assist daily mobility analysis. The main interface consists of a tablet integrating a movement-based interaction technique to dynamically explore time varying data. Even though the timewheel was outperformed by every other animation technique, the timeline controlled by our method was proven to be as efficient as the traditional animation (i.e. via mouse interaction), while being more engaging. Using touch to control animation was equally enjoyed by users, thus we combined physical and touch to provide more control and engagement to explore the indicators over time. Time exploration could benefit from the customizable dashboards approach. As an example, the user could view the same indicator at different time intervals side-by-side on the dashboard, as via small-multiples. Our prototype provides indicators of flows and displacements, as well as territory settlement dynamics. Despite not analyzing activities neither individual trajectories, it allows to understand how people move around on urban area. Our user studies intended to evaluate whether our design choices are useful to explore daily mobility, before fulfill the indicators.

Fifty-one volunteers, which 21 were knowledgeable on geography and mobility, used our prototype and have found it promising. Our approach is effective for assisting the visual analysis of daily mobility data (at least in the aggregate level). Participants could provide satisfactory answers to the proposed analytical questions, even those with none experience on the domain. The changes we made in our prototype from the pilot study to the evaluation phase improved efficiency, since the users answered 14 analytical questions in about $1 / 3$ the time the participants in the pilot study took to answer 12 questions with about the same accuracy $(80 \%)$.

We also interviewed a few public agents who explore HTS data to support decision-making in urban development. We demonstrate our prototype and let them test it. Though, they were excited with the idea of customizing the dashboard and using their own movement to explore time, they pointed out using a second screen (i.e. the tablet) could be a constraint in their everyday work, since it requires extra material, not needed for other tasks.

\section{CONCLUSION AND FUTURE WORK}

This paper proposes a geovisualization approach to support the (1) description of daily mobility through flows and displacements, territory settlement dynamics and individual trajectories; (2) analysis and comparison of complementary indicators in the aggregate and individual levels; (3) exploration of the time dynamics of indicators, and; (4) custom composition of the dashboard according to the user's needs, while choosing the spatial and temporal granularity to compose the indicators. eSTIMe uses a multi-screen environment embedding customizable dashboards, and a movement-based interaction to support time animation via mobile devices.

In a prototype level, it is limited by not including yet indicators on activities and individual trajectories. The exploration of the latter, while performed via survey data would not violate the data protection laws, since the spatial locations are defined by great areas, not involving specific geographical coordinates revealing individuals residences and work places, for instance. Usability issues are being solved towards a userfriendly environment. The evaluation process could demonstrate the efficacy of our approach, yet it does not evaluate the 
effectiveness of using customizable dashboards in comparison to the traditional ones. Therefore, future work also includes user studies to evaluate this aspect, and a validation of eSTIMe by domain experts.

\section{REFERENCES}

[1] D. J. Peuquet, "It's About Time: A Conceptual Framework for the Representation of Temporal Dynamics in Geographic Information Systems," Annals of the Association of American Geographers, vol. 84, no. 3, pp. 441-461, 1994.

[2] N. R. Council, Key transportation indicators: Summary of a workshop. Division of Behavioral and Social Science and Education. Washington, DC: National Academy Press., 2002.

[3] N. Andrienko, G. Andrienko, and H. Voss, Exploratory Analysis of Spatial and Temporal Data: A Systematic Approach. Springer Publishing Company, Incorporated, 2016.

[4] Y. Yan, Y. Tao, J. Xu, S. Ren, and H. Lin, "Visual analytics of bikesharing data based on tensor factorization," Journal of Visualization, vol. 21, no. 3, pp. 495-509, 2018.

[5] X. Shi, Z. Yu, J. Chen, H. Xu, and F. Lin, "The visual analysis of flow pattern for public bicycle system," Journal of Visual Language and Computing, pp. 1-9, 2017.

[6] G. Le Roux, J. Vallée, and H. Commenges, "Social segregation around the clock in the Paris region (France)," Journal of Transport Geography, vol. 59, pp. 134-145, 2017.

[7] E. Cochey and K. Tabaka, "Modes de représentation des trajectoires quotidiennes des habitants," in Huitièmes Rencontres de Théo Quant, Besançon, France, 2007, aCTN.

[8] A. Thudt, D. Baur, and S. Carpendale, "Visits : A Spatiotemporal Visualization of Location Histories," EuroVis, p. 2312, 2013.

[9] H. Otten, D. Marian, and M. Boris, "Are there networks in maps? An experimental visualization of personal movement data," In Personal Visualization: Exploring Data in Everyday Life, pp. 5-7, 2015.

[10] L. Shi, T. Jiang, Y. Zhao, X. Zhang, and Y. Lu, "UrbanFACET: Visually Profiling Cities from Mobile Device Recorded Movement Data of Millions of City Residents," arXiv preprint arXiv:1707.04210, 2017.

[11] C. Tominski, H. Schumann, G. Andrienko, and N. Andrienko, "Stackingbased visualization of trajectory attribute data," IEEE Trans. on Visualization and Computer Graphics, vol. 18, no. 12, pp. 2565-2574, 2012.

[12] J. S. Yi, Y.-a. Kang, J. T. Stasko, and J. A. Jacko, "Understanding and characterizing insights: How do people gain insights using information visualization?" in Proceedings of the 2008 Workshop on BEyond Time and Errors: Novel evaLuation Methods for Information Visualization, ser. BELIV '08. New York, NY, USA: ACM, 2008, pp. 4:1-4:6.

[13] M. Lu, Z. Wang, and X. Yuan, "TrajRank: Exploring travel behaviour on a route by trajectory ranking," IEEE Pacific Visualization Symp., vol. 2015-July, no. July, pp. 311-318, 2015.

[14] M. Lu, J. Liang, Z. Wang, and X. Yuan, "Exploring OD patterns of interested region based on taxi trajectories," Journal of Visualization, vol. 19, no. 4, pp. 811-821, 2016.

[15] Y. Wang, "A Visual Analysis System for Exploring Passenger Flow Information at Multiple Levels," in 2016 IEEE 20th International Conference on Computer Supported Cooperative Work in Design (CSCWD), 2016, pp. 363-368.

[16] X. Zhang and Q. Wang, "Peoplevis: A visual analysis system for mining travel behavior," in 2017 IEEE 21st International Conference on Computer Supported Cooperative Work in Design (CSCWD), 2017, pp. $463-468$.

[17] W. Zeng, C. W. Fu, S. M. Arisona, S. Schubiger, R. Burkhard, and K. L. $\mathrm{Ma}$, "Visualizing the Relationship Between Human Mobility and Points of Interest," IEEE Trans. on Intelligent Transportation Systems, vol. 18, no. 8, pp. 2271-2284, 2017.

[18] Y. Ma, T. Lin, Z. Cao, C. Li, F. Wang, and W. Chen, "Mobility Viewer: An eulerian approach for studying urban crowd flow," IEEE Trans. on Intelligent Transportation Systems, vol. 17, no. 9, pp. 2627-2636, 2016

[19] W. Zeng, C. W. Fu, S. Müller Arisona, A. Erath, and H. Qu, "Visualizing waypoints-constrained origin-destination patterns for massive transportation data," Computer Graphics Forum, vol. 35, no. 8, pp. 95-107, 2016.

[20] W. Wu, Y. Zheng, N. Cao, H. Zeng, B. Ni, H. Qu, and L. M. Ni, "MobiSeg: Interactive region segmentation using heterogeneous mobility data," in 2017 IEEE Pacific Visualization Symp. (PacificVis), 2017, pp. $91-100$.
[21] S. Few and P. Edge, "Dashboard confusion revisited," Perceptual Edge, pp. 1-6, 2007.

[22] A. J. Fischer, "Developing and evaluating a geographic information dashboard to improve spatial task performance," Ph.D. dissertation, Kansas State University, Department of Geography, 2018.

[23] L. Chittaro, "Visualizing information on mobile devices," Computer, vol. 39, no. 3, pp. 40-45, 2006.

[24] R. Langner, U. Kister, and R. Dachselt, "Multiple coordinated views at large displays for multiple users: Empirical findings on user behavior, movements, and distances," IEEE Trans. on Visualization and Computer Graphics, vol. 25, no. 1, pp. 608-618, 2019.

[25] U. Kister, K. Klamka, C. Tominski, and R. Dachselt, "GraSp: Combining Spatially-aware Mobile Devices and a Display Wall for Graph Visualization and Interaction," Computer Graphics Forum, vol. 36, no. 3, pp. 503-514, 2017.

[26] M. Pahud, E. Ofek, N. H. Riche, C. Hurter, and J. Grubert, "Mobiles as Portals for Interacting with Virtual Data Visualizations," arXiv preprint arXiv:1804.03211, 2018.

[27] K. Pietroszek, J. R. Wallace, and E. Lank, "Tiltcasting: 3D Interaction on Large Displays Using a Mobile Device," in Proceedings of the 28th Annual ACM Symp. on User Interface Software \&\#38; Technology, ser. UIST '15. New York, NY, USA: ACM, 2015, pp. 57-62.

[28] L. Besançon, P. Issartel, M. Ammi, and T. Isenberg, "Hybrid tactile/tangible interaction for 3d data exploration," IEEE Trans. on Visualization and Computer Graphics, vol. 23, no. 1, pp. 881-890, 2017.

[29] M. Baglioni, J. Macedo, C. Renso, and M. Wachowicz, "An ontologybased approach for the semantic modelling and reasoning on trajectories," in Advances in Conceptual Modeling - Challenges and Opportunities, 2008, pp. 344-353.

[30] P. Costa, G. M. Neto, and A. Bertolde, "Urban Mobility Indexes: A Brief Review of the Literature," Transportation Research Procedia, vol. 25, pp. 3645 - 3655, 2017, world Conference on Transport Research WCTR 2016 Shanghai. 10-15 July 2016.

[31] M. G. McNally and C. Rindt, "The activity-based approach," in Handbook of Transport Modelling, vol. 1, 2008, pp. 55-73.

[32] B. Bach, P. Dragicevic, D. Archambault, C. Hurter, and S. Carpendale, "A Descriptive Framework for Temporal Data Visualizations Based on Generalized Space-Time Cubes," Computer Graphics Forum, 2016.

[33] J. G. Grandi, H. G. Debarba, I. Bemdt, L. Nedel, and A. Maciel, "Design and assessment of a collaborative 3D interaction technique for handheld augmented reality," in 2018 IEEE Conference on Virtual Reality and 3D User Interfaces (VR), 2018, pp. 49-56.

[34] B. Pagno, D. Costa, L. Guedes, C. D. S. Freitas, and L. Nedel, "Guidelines for designing dynamic applications with second screen," in 2015 XVII Symp. on Virtual and Augmented Reality, 2015, pp. 42-51.

[35] M. Arvola and A. Holm, "Device-orientation is more engaging than drag (at least in mobile computing)," in Proceedings of the 8th Nordic Conference on Human-Computer Interaction: Fun, Fast, Foundational. ACM, 2014, pp. 939-942.

[36] A.-C. d. CEREMA [producer], "Enquête Ménages Déplacements (EMD), Grenoble, Grande Région Grenobloise," 2010.

[37] I. I. André-Poyaud, S. Chardonnel, L. L. Charleux, and K. Tabaka, "La mobilité au cœur des emplois du temps des citadins," in La mobilité qui fait la ville, 2008, pp. 67-95.

[38] J. Zhao, P. Forer, and A. S. Harvey, "Activities, ringmaps and geovisualization of large human movement fields," Information Visualization, vol. 7, no. 3-4, pp. 198-209, 2008.

[39] F. Bahoken, "Mapping flow matrices, a contribution," Theses, Université Paris Diderot (Paris 7), 2016.

[40] S. G. Hart and L. E. Staveland, "Development of NASA-TLX (Task Load Index): Results of empirical and theoretical research," in Human Mental Workload, 1988, vol. 52, pp. 139 - 183.

[41] J. Brooke et al., "SUS - A quick and dirty usability scale," Usability evaluation in industry, vol. 189, no. 194, pp. 4-7, 1996.

[42] J. R. Lewis, B. S. Utesch, and D. E. Maher, "UMUX-LITE: When there's no time for the sus," in Proceedings of the SIGCHI Conference on Human Factors in Computing Systems, ser. CHI '13. New York, NY, USA: ACM, 2013, pp. 2099-2102. 